ALBERT O. HIRSCHMAN 



\section{Albert O. Hirschman}

AN INTELLECTUAL BIOGRAPHY

Michele Alacevich 


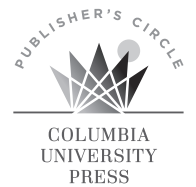

Columbia University Press gratefully acknowledges the generous support for this book provided by Publisher's Circle member Harriet Zuckerman.

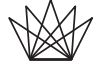 \\ Columbia University Press \\ Publishers Since 1893 \\ New York Chichester, West Sussex \\ cup.columbia.edu \\ Copyright () 2021 Michele Alacevich \\ All rights reserved
}

Library of Congress Cataloging-in-Publication Data

Names: Alacevich, Michele, author.

Title: Albert O. Hirschman : an intellectual biography / Michele Alacevich.

Description: New York City : Columbia University Press, 2021. |

Includes bibliographical references and index.

Identifiers: LCCN 2020030648 (print) | LCCN 2020030649 (ebook) |

ISBN 9780231199827 (hardback) | ISBN 9780231553308 (ebook)

Subjects: LCSH: Hirschman, Albert O. | Economists-United States-Biography. |

Economics-History.

Classification: LCC HB119.H57 A53 2021 (print) | LCC HB119.H57 (ebook) |

DDC $330.092[\mathrm{~B}]-\mathrm{dc} 23$

LC record available at https://lccn.loc.gov/2020030648

LC ebook record available at https://lccn.loc.gov/2020030649

Columbia University Press books are printed on permanent and durable acid-free paper.

Printed in the United States of America

Cover Design: Julia Kushnirsky

Cover Image: Albert O. Hirschman, 1962. Photograph by Hernán Díaz.

Courtesy of Katia Salomon 
To Elizabeth, my first reader and critic 

Reformers ... behave like the country or the chessplayer who exasperatingly fights on when "objectively" he has already lost-and occasionally goes on to win!

ALBERT HIRSCHMAN, 1963 
